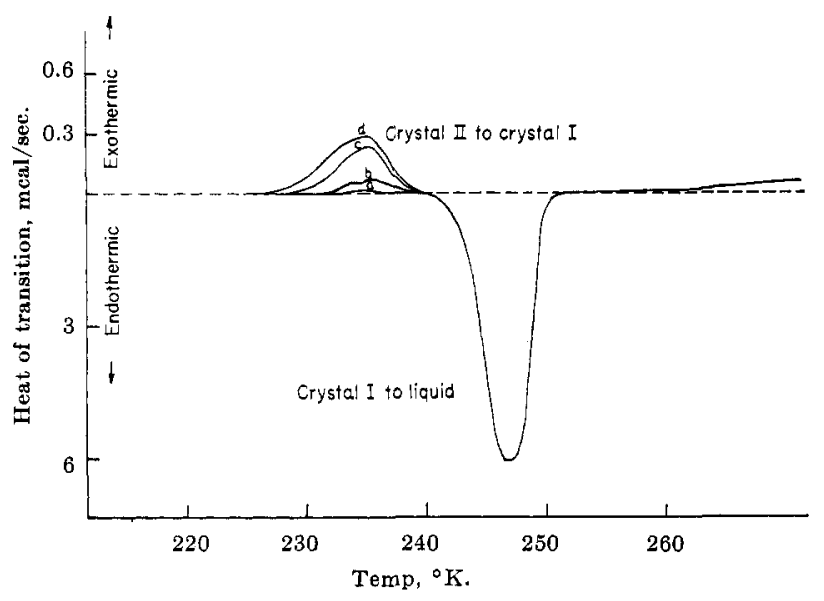

Figure 1. Differential scanning calorimetric thermogram of liquid glutaronitrile solidified by cooling: (a) at $2.5^{\circ} / \mathrm{min}$; (b) at $20 \% \mathrm{~min}$; (c) at $30 \% / \mathrm{min}$; and (d) by quenching on liquid nitrogen.

These ranged from $244 \mathrm{cal} / \mathrm{mole}$ for samples quenched in liquid nitrogen to $134,65,12,18$, and $1.5 \mathrm{cal} / \mathrm{mole}$ at cooling rates of $30,20,10,5$, and $2.5 \% \mathrm{~min}$, respectively. We failed to observe any reversibility in the transition from crystal II to crystal I.

Calculations by Wulff and Westrum indicate that the vibrational and rotational contributions to entropy are greater for the TG than the GG conformation. $^{5}$ The observed exothermic transitions from metastable crystal II to crystal I, which is in the GG conformation, ${ }^{2,5}$ thus give additional support to the assignment ${ }^{2}$ of the TG conformation to the metastable crystal. However, the assignment of the metastable modification solely to the TG conformation remains to be established, in view of the dependence of the exothermic heats on the cooling rate.

Acknowledgment. Acknowledgment is made to the donors of The Petroleum Research Fund, administered by the American Chemical Society, for support of this research.

\section{A Note on the Principle of Corresponding States}

by F. Danon and J. C. Rossi

Facultad de Ciencias Exactas y Naturales, University of Buenos Aires, Buenos Aires, Argentina (Received August 20, 1965)

As the nature of the molecular interactions in argon, krypton, and xenon satisfies the restrictive conditions required by the statistical-mechanical derivation of the law of corresponding states, ${ }^{1}$ their thermodynamic properties have extensively been used to test the validity of this law. ${ }^{2}$ Reducing factors are derived from both critical constants and molecular parameters. ${ }^{2-4}$ The properties considered in this note are the second virial coefficient and the viscosity coefficient at low pressure reduced in terms of the parameters of the Kihara pair potentia $]^{5} U=U_{0}\left[\left(\rho_{0} / \rho\right)^{12}-\right.$ $\left.2\left(\rho_{0} / \rho\right)^{6}\right]$, where $U_{0}$ is the minimum of the potential which occurs at $\rho_{0}$. The intermolecular separation $\rho$ is defined as the shortest distance between the outer surface of the cores. The given equation is valid for $\rho \geqslant d$, where $d$ is the core diameter. For smaller values of $\rho, U \geqslant \infty$. The reduced expression for the second virial coefficient $B$ is then $B^{*}\left(T^{*}\right)=B\left(T^{*}\right) /$ $2 \pi / 3 \rho_{0}{ }^{3}$, where $T^{*}=T k / U_{0}$. Myers and Prausnitz ${ }^{6}$ have made a similar study for the second virial coefficient, but the experimental data now available, mainly at low temperatures, ${ }^{7}$ allow a more precise check on the corresponding states behavior. No transport property has previously been studied on a corresponding states basis, although calculations on argon only have been done by Barker, et al. ${ }^{8}$ The core size we use is not an extra adjustable parameter but is independently fixed by the equation $X=7.0 \omega+$ 0.24 , where $X$ is a core parameter related to the mean curvature, $M_{0}$, of the core, and $\omega$ is the acentric factor introduced by Pitzer. ${ }^{10}$ The heavy rare gases (Ar, $\mathrm{Kr}, \mathrm{Xe}$ ) have $\omega=0$ and for a spherical core $M_{0}=2 \pi d$, so that the simple relationship $d=0.08 \rho_{0}$ results. We thus obtain a two-parameter potential function as required by the theory. ${ }^{1}$

Parameters were obtained by simultaneous fitting of both second virial and viscosity coefficient data by automatic computation. We minimized the root mean square deviation between theoretical and experimental values of both properties. Results are shown in Table I and full details are given in ref 11.

Although the general quality of the Kihara potential was studied elsewhere, ${ }^{11}$ the theoretical curve is drawn

(1) K. S. Pitzer, J. Chem. Phys., 7, 583 (1939).

(2) J. O. Hirschfelder, C. F. Curtiss, and R. B. Bird, "Molecular Theory of Gases and Liquids," John Wiley and Sons, Inc., New York, N. Y., 1954.

(3) J. M. H. Levelt, Physica, 26, 361 (1960).

(4) F. Danon and K. S. Pitzer, J. Phys. Chem., 66, 583 (1962).

(5) T. Kihara, Rev. Mod. Phys., 25, 839 (1953).

(6) A. L. Myers and J. M. Prausnitz, Physica, 28, 303 (1962).

(7) B. E. Fender and D. Halsey, J. Chem. Phys., 36, 1881 (1963).

(8) J. A. Barker, W. Fock, and F. Smith, Phys. Fluids, 7, 897 (1964).

(9) F. Danon and K. S. Pitzer, J. Chem. Phys., 36, 425 (1962).

(10) K. S. Pitzer, et al., J. Am. Chem. Soc., 77, 3433 (1955).

(11) J. C. Rossi and F. Danon, Discussions Faraday Soc., in press. 


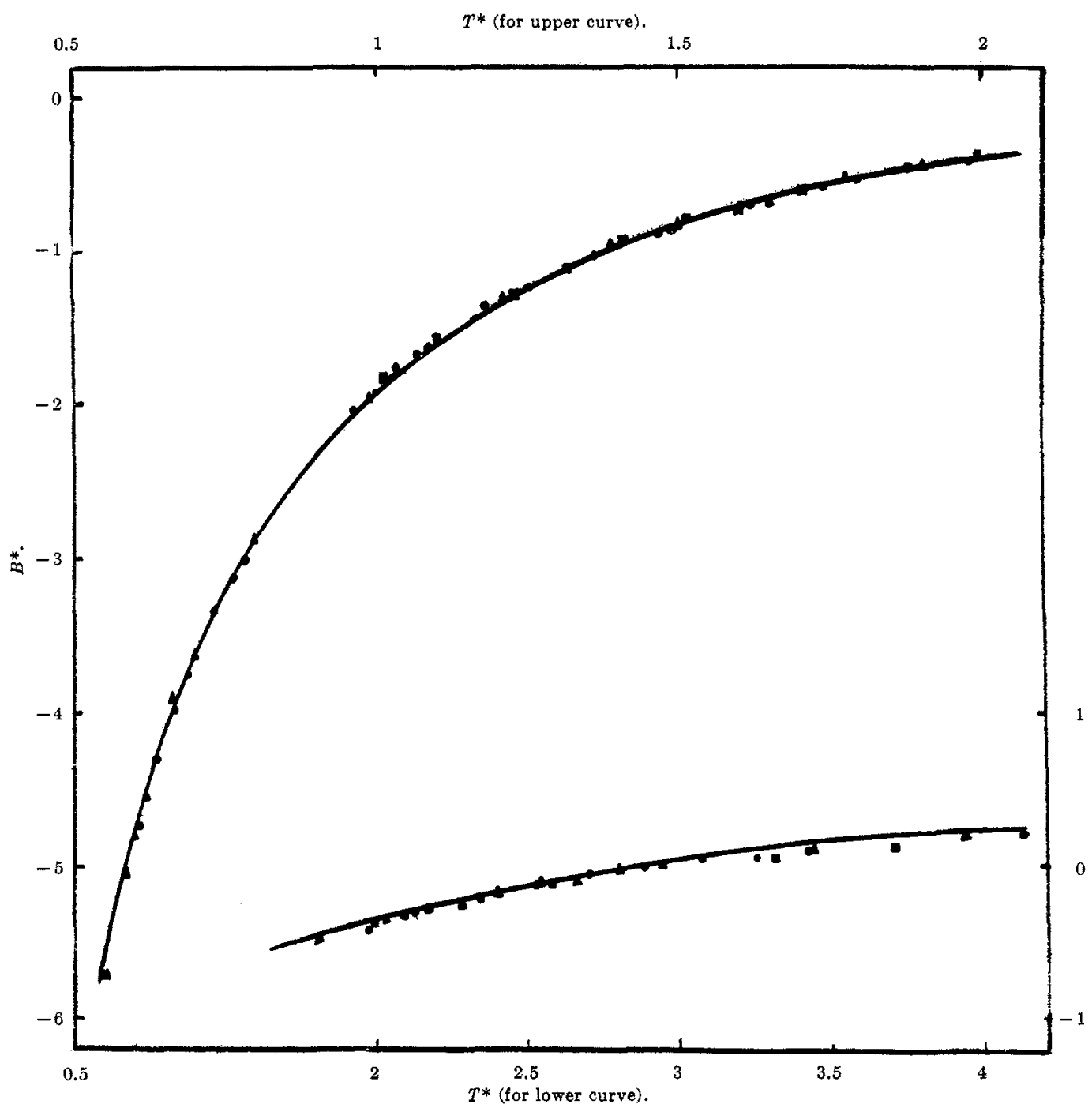

Figure 1. Reduced second virial coefficient: $\bullet$, argon; $\mathbf{\Delta}$, krypton; $\mathbf{\square}$, xenon.

Table I: Parameters of the Kihara Pair Potential

\begin{tabular}{|c|c|c|c|c|c|}
\hline & $\begin{array}{c}U_{0} / k \\
{ }^{\circ} \mathrm{K}\end{array}$ & Ao, & $\begin{array}{l}d, \\
\text { A }\end{array}$ & $\begin{array}{l}\text { Standard } \\
\eta \times 10^{7} \\
(m T)^{1 / 2}\end{array}$ & $\begin{array}{c}\text { deviations } \\
B(T), \\
A^{2} / \text { mole- } \\
\text { cule }\end{array}$ \\
\hline Argon & 138.0 & 3.482 & 0.279 & 0.39 & 1.60 \\
\hline Krypton & 196.1 & 3.735 & 0.299 & 0.07 & 3.9 \\
\hline Xenon & 263.6 & 4.078 & 0.326 & 0.03 & 1.1 \\
\hline
\end{tabular}

in Figure 1 to show the excellent agreement with the experiment over the entire range of temperature of $B^{*}\left(T^{*}\right)$. Figure 1 clearly shows that the corresponding states behavior is closely followed by the second virial coefficient of $\mathrm{Ar}, \mathrm{Kr}$, and $\mathrm{Xe}$. The reduced viscosity coefficient $\eta^{*}=\eta R_{0}^{2} / \sqrt{m U_{0}}$ is shown in Figure 2. Here $R_{0}=2^{-1 / 6} \rho_{0}+d$ is the collision diameter and $m$ is the mass of the molecule. The recent data on $\mathrm{Kr}$ are included $;^{12}$ this allows a comparison on a larger temperature range than was previously possible. ${ }^{2}$ The agreement between theory and experiment is again very good and it is seen that the principle of corresponding states is also followed by the property. Levelt ${ }^{3}$ has made a careful study of the compressibility of argon and xenon and concluded that it is impossible to represent the experimental $B-T$ curve of argon and xenon by means of a Lennard-Jones 6-12 potential within experimental accuracy. These curves however can be brought into coincidence by means of two reduction factors for temperature and density. The $P V / R T$ isotherm for argon may also be transformed into the compressibility isotherm of xenon by multiplying by

(12) D. G. Clifton, J. Chem. Phys., 38, 1123 (1963). 


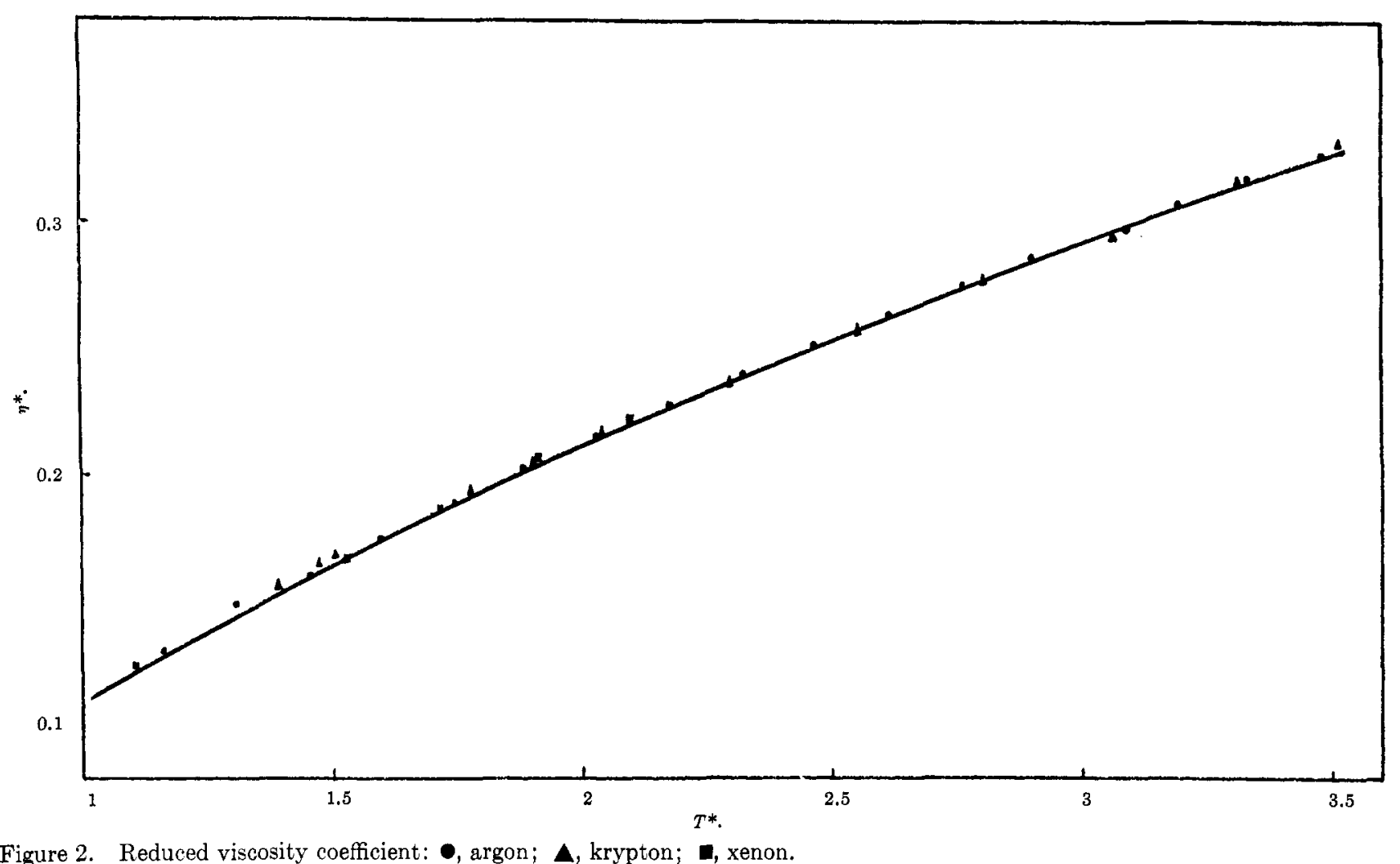

scale factors. Levelt found the "ideal scale factors", 3 i.e., best set of scale factors, to be 1.931 for the temperature and 0.633 for the density. The ratios of the reduction factors in terms of the molecular parameters are shown in Table II.

Table II: Ratio of Reduction Factors

\begin{tabular}{|c|c|c|c|c|c|c|}
\hline & \multicolumn{2}{|c|}{$\overbrace{\text { L-J } 6-12}^{\text {Levelt- }}$} & \multicolumn{2}{|c|}{ Present work } & \multicolumn{2}{|c|}{ —"Ideal"- } \\
\hline & Temp & Density & Temp & Density & Temp & Density \\
\hline$\frac{\text { Param Xe }}{\text { Param Ar }}$ & 1.889 & 0.595 & 1.910 & 0.623 & 1.931 & 0.633 \\
\hline
\end{tabular}

It is seen that the ratios we obtain are much closer to the ideal ones than Levelt's. In order to obtain coincidence of the reduced isotherms, one should modify one set of parameters by about $1 \%$ in the temperature scale factor and by $0.6 \%$ in the density scale factor, values which are scarcely larger than the expected uncertainties of the parameters. Consequently, if the assumption of the pairwise additivity of the potential is valid, one expects that if the isotherms of argon and xenon are reduced with our set of molecular parameters they should come much closer to each other than if reduced with the Lennard-Jones 6-12 potential parameters, so that the compressibility factor of these gases can also be correlated using the principle of corresponding states.

\section{Effect of Solvent on the Entropy of the}

Tris-(1,10-phenanthroline)iron(III)-(II) System

by Byron Kratochvil and John Knoeck

Department of Chemistry, University of Wisconsin, Madison, Wisconsin 59706 (Received August 30, 1965)

The partial molal entropy change occurring during a reversible electron transfer in solution can be obtained experimentally through measurement of a cell potential as a function of temperature. A compilation of temperature coefficients for a number of aqueous electrode potentials has been made by deBethune and Loud. ${ }^{1}$ These data indicate that for systems in which the

(1) A. J. deBethune and N. S. Loud, "Standard Aqueous Electrode Potentials and Temperature Coefficients at $25^{\circ} \mathrm{C}, " \mathrm{C}$. A. Hampel, Publisher, Skokie, Ill., 1964. 